\title{
Requirement Engineering - Analysis of Risk with Goal Method
}

\author{
Shankarnayak Bhukya \\ Associate professor \\ SVIT,Secunderabad \\ Hydaerabad,Telangana,India
}

\author{
Suresh Pabboju, PhD \\ Professor,C.B.I.T \\ Osmania University \\ Hydaerabad,Telangana,India
}

\begin{abstract}
Requirement Engineering says that "Requirements definition" is a careful assessment of the need that a system is to fulfill that must say why a system is based on current external market. A good analysis of the domain is extremely important for the success of the system that understanding the needs and motivations of stakeholders and analyzing the complex social relationship help in coming up with the correct requirement for the system. A requirement is a goal achievement is the responsibility of a single software agent assumption is a goal whose achievement is delegated to a single agent in the environment unlike requirements expectations cannot be enforced by the software to be satisfied to organizational regulations. Our research is integration of ERP modules requirement analysis is to identify with Goal model is used to provide the basis for detection and management conflicts capture variability in the problem domain through the use of alternative refinements and alternative assignment. The analysis result list of problems and deficiencies that can be precisely formulated and calculated risk using Tropos Goal layer.
\end{abstract}

\section{Keywords}

Requirement Engineering, Tropos Model, Risk Analysis, ERP Integration, Genetic algorithm.

\section{INTRODUCTION}

The term risk is defined as the potential future harm that may arise due to some present actions as explained in Wikipedia. Risk management in software engineering is related to the various future harms that could be possible on the software due to some minor or non-noticeable mistakes in software development project or process. "Software projects have a high probability of failure so effective software development means dealing with risks adequately (www.thedacs.com)." Risk management is the most important issue involved in the software project development. This issue is generally managed by Software Project Management (SPM). During the life cycle of software projects, various risks are associated with them. These risks in the software project is identified and managed by software risk management which is a part of SPM. Some of the important aspects of risk management in software engineering are software risk management, risk classification and strategies for risk management. Requirements engineering is a process based method for defining, realizing, modeling, relating, documenting and maintaining software requirements in software lifecycle that help to understand the problem better. It has been shown that a large proportion of the

Publications in software development can be related back to requirements engineering (RE).

$\mathrm{RE}$ is the process of discovering the purpose in the software development, by identifying stakeholders and their needs, and documenting these in a form that is amenable to analysis, communication and subsequent implementation. Failures during the Requirement Engineering procedure have a significant negative impact on the overall development process. Reworking requirements failures may take $40 \%$ of the total project cost. If the requirements errors are found late in the development process, e.g. during maintenance, their correction can cost up to 200 times as much as correcting them during the early stages of the development process. Adequate necessities are therefore essential to ensure that the system the customer expects is produced and that unnecessary exertions are avoided. To avoid risk factors in software development optimization of Requirements Engineering analysis leads to substitute sets of functional necessities posed by stakeholders, goal-oriented approach for analyzing risks during the requirements analyzed with stakeholder interests and then countermeasures are identified and as part of the systems requirement.

The main factors that a requirement engineering process considers are business requirements and user requirements. The requirements are used to enhance the development of the software product with low cost and the time it should satisfy all the requirements. One of the sensitive areas, which every software development process concentrate is the risk involved with the process. So, particular assessment measures have to be taken in order to minimize the risks in software development process. YudistiraAsnarand Paolo Giorgini have proposed security risk analysis in requirement engineering. The method deals with a software development method called, Goal Model and with a Probabilistic Risk Analysis (PRA).Inspired from their work, we are planning to propose security in risk analysis on extending the model with risk analysis feature. 


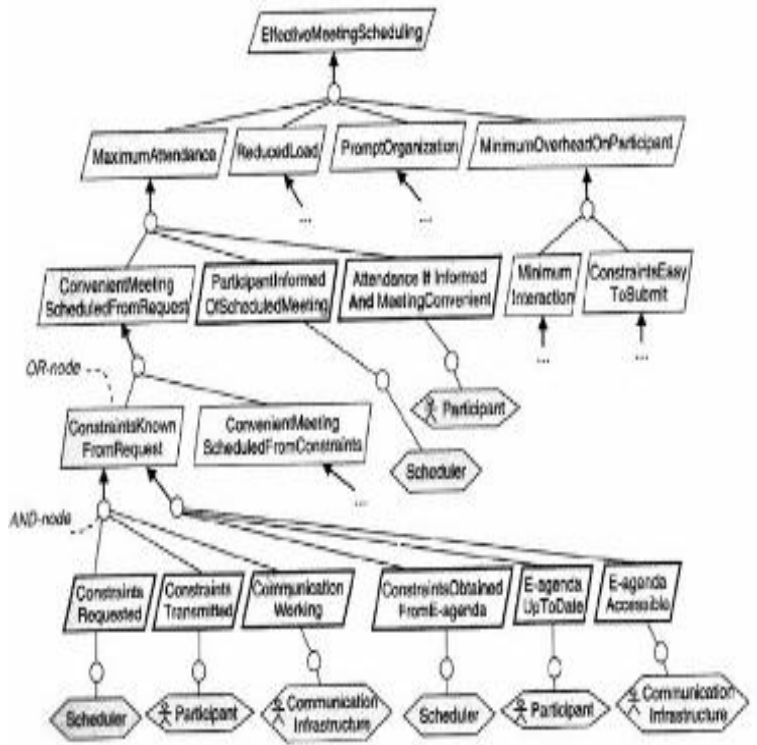

Figure 1 Represents the Risk Analysis Model

Goal-based methods consider the views towards the system its stakeholder's goal needed to achieve the goals. The software to be developed is only as needed to achieve the goals. On the one hand a hospital wants to provide treatment for a patient to administer. Researcher wants to use this monitoring data to conduct a medical study against the privacy goals of the patient. Goal model provide the means to detect and resolve the conflicts of overall system is significant because the system to be only modeled in some artifacts have relation to a goal. The problem based methods follow different approach solve by the system environment allow one to split the problem into sub-problems interaction with its environment further refined into a design description and a system architecture method.

\section{RELATED WORK}

Since there could be various risks associated with the software development projects, the key to identify and manage those risks is to know about the concepts of software risk management. Many concepts about software risk management could be identified but the most important are risk index, risk analysis, and risk assessment (Hoodat, H. \& Rashidi, H.).Security risk assessment in the requirements phase is challenging because probability and damage of attacks is not always numerically measurable or available in the early phases of development. Selecting proper security solutions is also problematic because mitigating impacts and side-effects of solutions are not often quantifiable either. In the early development phases, analysts need to assess risks in the absence of numerical measures or deal with a mixture of quantitative and qualitative data. GolnazElahietal propose a risk analysis process which intertwines security requirements engineering with a vulnerability-centric and qualitative risk analysis method. The method is qualitative and vulnerabilitycentric, in the sense that by identifying and analyzing common vulnerabilities the probability and damage of risks are evaluated qualitatively. They also provided an algorithmic decision analysis method that considers risk factors and alternative security solutions, and helps analysts select the most cost-effective solution. The decision analysis method enables making a decision when some of the available data is qualitative. JacKyAnget al has developed an expert system that has least focus on requirement engineering. In facts, requirement engineering is important to get all the requirements needed for an expert system. If the requirements do not meet the clients need, the expert system is considered fail although it works perfectly. Lukas Pilatet introduced the problem in requirement engineering is the communication with backend attributed to the different languages spoken by these stakeholders based on their different background and domain knowledge. Related problem involved with transferring and sharing such knowledge when stakeholders are reluctant to take knowledge management process and adopt the concept of the spiral of knowledge involving transformations from tacit to explicit knowledge.

In order to develop a special aimed software classify combine and prioritize different requirements pre-requisites functional non-functional requirements engineering process they can classify the requirements. Development of special software requires different requirements to be categorized during software life cycle whether they are important and necessary for our development, at present time or they are not important currently but will become important in future. Requirements engineering aims to recognize the stockholders requirements and their verifications then gaining agreement on system requirements is not just a phase completed at the beginning of system development not required any more but includes parts of next phase of software development. The purpose of acquired a comprehensive knowledge about requirements development aim in the software production life cycle engineering activity is used to describe for a better comprehensive subject.

\section{PROBLEM DEFINITION}

The requirement analysis is a field in the software development are modelled according to the requirement of client and developed under requirement engineering will satisfy the client perspective. The requirement analysis based on requirement development processes are also challenged by the risks in developing the software, efficient risk analysis management system is inevitable for the software development process under requirement development. Goal Approach to reduce effort cost to model for tackling the risk associated with the software engineering modelled in three layers, the top layer is the goal layer event layer is second and support layer is the third layer. A method to analyze the association between the nodes of each layer to evaluate the chances of raising the risks analysis on the associations of three layers, this approach explores the parameter like security and denial to efficient analysis of the risk factor.

\subsection{Software Risk Management:}

Since there could be various risks associated with the software development projects, the key to identify and manage those risks is to know about the concepts of software risk management. Many concepts about software risk management could be identified but the most important are risk index, risk analysis, and risk assessment (Hoodat, H. \& Rashidi, H.).

Generally risks are categorized into two factors namely impact of risk events and probability of occurrence. Risk index is the multiplication of impact and probability of occurrence. Risk index can be characterized as high, medium, or low depending upon the product of impact and occurrence. Risk index is very important and necessary for prioritization of risk (Hoodat, $\mathrm{H}$. \& Rashidi, H.)

\subsubsection{Risk Analysis}

There are quite different types of risk analysis that can be used. Basically, risk analysis is used to identify the high risk elements of a project in software engineering. Also, it 
provides ways of detailing the impact of risk mitigation strategies. Risk analysis has also been found to be most important in the software design phase to evaluate criticality of the system, where risks are analyzed and necessary counter measures are introduced (Hoodat, H. \& Rashidi, H.). The main purpose of risk analysis is to understand risks in better ways and to verify and correct attributes. A successful risk analysis includes important elements like problem definition, problem formulation, data collection (Hoodat, H. \& Rashidi, H.).

\subsubsection{Risk Assessment:}

Risk assessment is another important case that integrates risk management and risk analysis. There are many risk assessment methodologies that focus on different types of risks. Risk assessment requires correct explanations of the target system and all security features (Hoodat, H. \& Rashidi, H.). It is important that a risk referent levels like performance, cost, support and schedule must be defined properly for risk assessment to be useful.

The key purpose of classifying risk is to get a collective viewpoint on a group of factors. These are the types of factors which will help project managers to identify the group that contributes the maximum risk. A best and most scientific way of approaching risks is to classify them based on risk attributes. Risk classification is considered as an economical way of analyzing risks and their causes by grouping similar risks together into classes (Hoodat, H. \& Rashidi, H.). Software risks could be classified as internal or external. Those risks that come from risk factors within the organization are called internal risks whereas the external risks come from out of the organization and are difficult to control. Internal risks are project risks, process risks, and product risks. External risks are generally business with the vendor, technical risks, customers' satisfaction, political stability and so on. In general, there are many risks in the software engineering which is very difficult or impossible to identify all of them. Some of most important risks in software engineering project are categorized as software requirement risks, software cost risks, software scheduling risk, software quality risks, and software business risks. During the software development process various strategies for risk management could be identified and defined according to the amount of risk influence. Based upon the amount of risk influence in software development project, risk strategies could be divided into three classes namely careful, typical, and flexible. Generally, careful risk management strategy is projected for new and inexperienced organizations whose software development projects are connected with new and unproven technology; typical risk management strategy is well-defined as a support for mature organizations with experience in software development projects and used technologies, but whose projects carry a decent number of risks; and flexible risk management strategy is involved in experienced software development organizations whose software development projects are officially defined and based on proven technologies.

\section{CASE STUDY}

One of the phases in Software development is requirement analysis that considers business requirement and user requirement used to enhance the development of the software product with low cost and in the time \& effort it should satisfy all the requirements to every software process. The requirement engineering is also affected by the risk factors of the software based on user requirements has higher risk factors and also the security factors proposed by Tropos. A goal relation model is to find the frequent goals to achieve the risk and cost to achieve the same.

The case study includes the processing in a software product for achieving maximum profit proposed the development of software product with ERP modules. This case study is subjected to analyze the cost required to reach a target and the risk that can happen during the different level of the processing. The aim of the three main features considered in the proposed case study is to earn money with less effort and risk from the services. The case study is proposed by considering the scenario like a client want to develop software from the organization. In depth analysis of different process happening in developing software detailed in the case study several uncertain events happen will restrict the successful achievements measures to be taken to reduce the effect of these uncertain events happen costs for the whole process and they should be analyzed carefully before adoption.

\subsection{Tropos Model in Requirement Engineering}

Tropos goal model is a software model which is characterized by concepts of agent goal task and resource and uses them throughout the development process from requirements analysis to implement. Requirement analysis model provides the organizational settings where the system to be eventually operate extended by adding constraints and relation in order to assess the risk factor[4]. The tropos GR model consist of three tuples the number of node $(\mathrm{N})$ number of relations $(\mathrm{R})$ and uncertain events ( $\mathrm{U}$ ) and risk model (GR) which supports three layers namely goal layer event layer and support layer that needs have to be achieved[5]. The event layer serves to achieve the goals with nodes, the support layer contains node which supports either the event nodes or goal nodes. Each of the three constraints is characterized by severity value and the severity is marked with four measure strong positive, positive, negative and strong negative, the attributes of normal and abnormal represented by NOR (c) and abnormal (c ) where c is to construct either goals events and supports evidence of construct $\mathrm{c}$ will be normal for NOR ( $\mathrm{s}$ ) and $\mathrm{ABN}$ ( $\mathrm{s}$ ), the probability theory if Prob ( A ) $=0.1$ then we can infer that probability of $-\mathrm{A}$ is 0.9 evidence of a goal being abnormal $(\mathrm{ABN})$ cannot be inferred from evidence on the satisfaction of the goal (NOR)[6]. For object the software development company has the goal to develop called business development software which is affected by the event that needs to be given importance called procurement of raw materials[7]. The event may trigger the goal to either NOR () or to ABR () according to the support value, if the support user requirement has severity then the goal result in $\mathrm{ABN}$ (). The attribute values are specified more clearly by representing the value in different range like fully ( $\mathrm{f}$ ) partially $(\mathrm{p})$ and none $(\mathrm{n})$ and the priority of those values like $f>p>n$, evidence for the satisfaction of a goal means that there is at least sufficient evidence to support the claim that the goal will be fulfilled. 


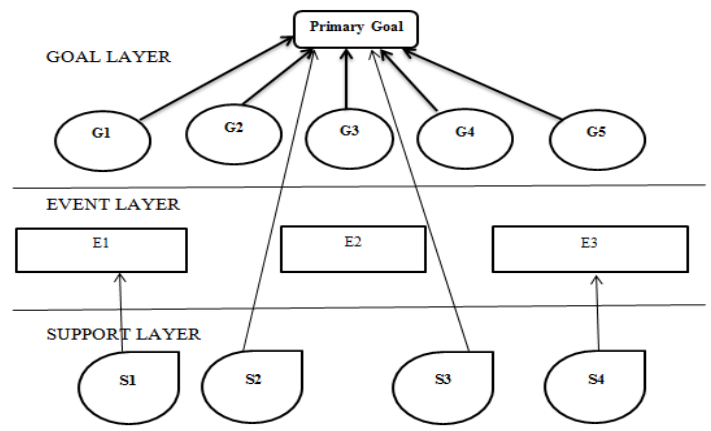

Figure 2 the goal model of requirement analysis

The relation $\mathrm{R}$ is the relations defined over different nodes in the defined goal risk model can be represented as $R=\left[N_{1}, \ldots\right.$. $\mathrm{N}_{\mathrm{n}}>\mathrm{N}$ ] where $\mathrm{N}$ is the target node and the $\mathrm{N}_{1, \ldots .} \mathrm{N}_{\mathrm{n}}$ are the sources nodes decompose relation contribution relation and alleviation relation. The decompositions relations which used are $\mathrm{AND} / \mathrm{OR}$ for refining the goal event support relation points the impact of one node to another relations each one of these types can propagate either evidence for Nor or ABN or both. The ++ contribution relation indicates the relation propagates both NOR and $\mathrm{ABN}$ evidence and the ++ contribution relation means the relation only propagates NOR evidence toward target nodes. The model goal is associate goals are the main criteria behind the success of the main goal. The success rate is projected based on the cost to which the main goal is neither achieved with an acceptable risk, cost to risk analysis based on NOR value and $A B N$ value of the associated goal.

The top layer of model contains the target goals and associate goals which are needed for the projection of the goals supporting events are listed in the support layer as support values. Cost to risk estimation is conducted to find the best cost efficient way to calculate the target goal or achieve the goal describe the methodological process and qualitative risk reasoning techniques used to analyze and evaluate alternatives in a GR model. We focus on finding and evaluating all possible ways for satisfying top goals with an acceptable level of risk, given a GR model each OR-decomposition introduces alternatives modalities for top goal satisfaction different sets of leaf goals that can satisfy top goals.

\subsection{Genetic Algorithm for Optimizing the Dataset solutions}

Software product organization in the model of Tropos goal model, the top layer of model contains the target goals and associate goals event layer contains the associated events, which are needed for the projection of the goals supporting events are listed in the support layer as support values. Cost to risk estimation is conducted to find the best cost effective way to calculate the target goal or achieve the goal. Risk can be mitigated with appropriate countermeasure however may introduce additional costs and further complication are used for evaluating the different risks caused by different events based on the responses from the above list the risk are mitigated to an accepting level with feasible cost for development.

Risk analysis approach are used for evaluating the different risk caused by different events based on the responses from the above list analysis the risk are mitigated to an accepting level with feasible cost for development. Achieving the goal G1 can use anyone of the source nodes G2, G3 and G4 discussed here has 4 target nodes they are G5 G1 G6 and G9.
The different data set solutions for achieving the targets are represented with $\mathrm{S}[8]$.

Consider the following dataset solutions which are used for evaluating the target nodes.

\section{S1 - G2 G4 G7 G10 G11 G12}

\section{S2 - G2 G3 G8 G10 G11 G12}

S3 - G2 G4 G7 G8 G11 G12

S4 - G2 G4 G7 G10 G11 G12

S5 - G3 G4 G7 G10 G11 G12

S6 - G3 G4 G8 G10 G11 G12

S7 - G2 G3 G4 G7 G10 G12

\section{S8 - G2 G3 G4 G8 G11 G12}

The genetic algorithm is used to optimize algorithm data management domain, optimization method of GA in the proposed approach to generate the optimized dataset solutions. The data set solutions obtained from the Tropos goal model are considered as the initial population which is subjected for the optimization. The cost calculation is conducted as the fitness function genetic algorithms for dataset solution are formed of six source node value[10]. The cost analysis to extract the cost effective dataset solutions among the extracted dataset solutions, the impact of each process in the software development in the case of the SDC is obtained by

the association of the node to the source node value cost analysis is subjected to extract cost effective dataset among the extracted solutions, calculating the association of the node to the source node as a set of three tuples.

$\operatorname{Cost}\left(\mathrm{G}_{\mathrm{n})}=\left[\operatorname{Cost}\left(\mathrm{G}_{\text {source }}\right)\right.\right.$, Cost $($ En $)$ Cost $\left.(\operatorname{Sn})\right][11]$

The object $\mathrm{G}_{\mathrm{n}}$ means the cost of the $\mathrm{n}$ node consider $\mathrm{G}_{\text {source }}$ is the number of source goal node is supporting the target goal node values En and $\mathrm{Sn}$ are the cost for event nodes and the support node value is also affected by NOR values and ABN values of the nodes that are relevant for achieving the target goal. The factors are that affect the cost are the likelihood and severity value of the event node that support the evaluation of the data set is described in the below.

\section{S6 - G3 G8 G10 G11 G12}

The characteristic feature of the genetic algorithm to improve the stability of the population is the set of dataset solutions obtained after the fitness evaluation.[9] The operation is executed by selecting two data sets solutions from the population and interchanging the characters, point is set to cross the two dataset solutions and such point is termed as the crossover point, the lists of source goal layer are

$$
\begin{aligned}
& \mathrm{G} 3>\mathrm{SAT}(\mathrm{P}) \mathrm{E} 1(++)>\mathrm{S} 1(+)>\mathrm{E} 6(-)=10+5-5-10 ; \\
& \mathrm{G} 4>\mathrm{SAT}(\mathrm{F}) \mathrm{E} 1(++)>\mathrm{S} 1(+)>\mathrm{E} 6(+)=10+5+5=20 ; \\
& \mathrm{G} 8>\mathrm{SAT}(\mathrm{F}) \mathrm{E} 1(++)>\mathrm{S} 1(+)>\mathrm{E} 7(+)=10+5+5=20 ; \\
& \mathrm{G} 10>\mathrm{DEN}(\mathrm{F}) \text { E10 }(--)>\mathrm{S} 4(-)=0 ; \\
& \mathrm{G} 11>\mathrm{SAT}(\mathrm{P})=5 ; \\
& \mathrm{G} 12>\mathrm{DEN}(\mathrm{P})=5 ; \\
& \operatorname{COST}(\mathrm{S} 6) \quad=60 ;
\end{aligned}
$$

Candidate solutions are set for obtaining the cost value in achieving the target goals, calculating all the cost values are 
plotted and a threshold is fixed for the cost value. The threshold is set based on maximized SAT value and minimized DEN value selects the genetic algorithm processes fitness of each candidate solutions are calculated the GA subjects the two other processes.

Crossover process of the characteristic features of the genetic algorithm to improve the stability of the population set the candidate solutions from the population and interchanging there characters. A point is set to cross the two candidate solutions and such point is termed as the crossover point.

Mutation process is an associated process of the crossover process offspring generated after the crossover process are selected for the mutation process, the mutation process one point is randomly selected from the offspring and a new character is assigned to selected point by replacing the existing one[12]. Represents the process of mutating an offspring according to the definitions of genetic algorithm created by applying crossover and mutation over the initial population, feasible population is calculated based on the fitness evaluation function defined by the proposed approach.

\section{IMPLEMENTATION RESULTS}

The proposed system is case study of integration ERP modules involves in all the requirements like sales \& distribution, Finance \& Controlling, Material Management, Procurement and Production planning of software Development Company targeted to a prime goal. The requirements of these modules integrate with cost and effort to gain the profit on software product G1 and a number of goals are associated with G1 to make G1 achievable by the Software development cycle. The tropos goal model is defined over the software development cycle by considering the SAT and DEN values that are candidate solutions are generated tropos model of length 6 to 8 for the efficient evaluation of the cost and risk of the software development life cycle. The tropos goal assigned cost values by randomly generated program and the cost values are associated with the events and supports defined in the Tropos goal model of the SDC

Table 1 Cost\& Effort of Software development of ERP

\begin{tabular}{|l|l|l|l|l|l|}
\hline $\begin{array}{l}\text { Event } \\
\text { S }\end{array}$ & $\begin{array}{l}\text { value } \\
\text { S }\end{array}$ & $\begin{array}{l}\text { Goal } \\
\text { s }\end{array}$ & $\begin{array}{l}\text { cost } \\
\text { s }\end{array}$ & $\begin{array}{l}\text { support } \\
\text { s }\end{array}$ & $\begin{array}{l}\text { association } \\
\text { s }\end{array}$ \\
\hline E1 & 7 & G1 & 4 & S1 & 8 \\
\hline E2 & 7 & G2 & 5 & S2 & 0 \\
\hline E3 & 4 & G3 & 4 & S3 & 2 \\
\hline E4 & 7 & G4 & 6 & S4 & 4 \\
\hline E5 & 5 & G5 & 1 & S5 & 4 \\
\hline E6 & 0 & G6 & 5 & S6 & 2 \\
\hline E7 & 0 & G7 & 3 & S7 & 7 \\
\hline E8 & 2 & G8 & 6 & S8 & 9 \\
\hline E9 & 4 & G9 & 3 & S9 & 5 \\
\hline E10 & 1 & G10 & 2 & S10 & 5 \\
\hline
\end{tabular}

Based on above values of the tables the costs of the generated candidate solutions are calculated the genetic algorithm is applied to the generated candidate solutions according to number of solutions are extracted from the initial population as the cost effective solutions of the Software development.

Table 2 is Candidate solution for requirement analysis

\begin{tabular}{|c|}
\hline Candidate solutions \\
\hline$[\mathrm{G} 4, \mathrm{G} 7, \mathrm{G} 8, \mathrm{G} 9, \mathrm{G} 10]$ \\
\hline$[\mathrm{G} 4, \mathrm{G} 6, \mathrm{G} 7, \mathrm{G} 8, \mathrm{G} 9]$ \\
\hline$[\mathrm{G} 2, \mathrm{G} 7, \mathrm{G} 8, \mathrm{G} 9, \mathrm{G} 10]$ \\
\hline$[\mathrm{G} 3, \mathrm{G} 4, \mathrm{G} 5, \mathrm{G} 6, \mathrm{G} 7]$ \\
\hline$[\mathrm{G} 2, \mathrm{G} 3, \mathrm{G} 4, \mathrm{G} 5, \mathrm{G} 6, \mathrm{G} 7, \mathrm{G} 8]$ \\
\hline$[\mathrm{G} 2, \mathrm{G} 4, \mathrm{G} 5, \mathrm{G} 6, \mathrm{G} 7, \mathrm{G} 8, \mathrm{G} 9, \mathrm{G} 10]$ \\
\hline
\end{tabular}

From above analysis risk is defined to calculate the cost and relevant risk to the obtained candidate solutions, the cost to risk analysis phase candidate solution for achieving the target goals are analyzed. The risk calculated based on the DEN value of the candidate solutions under construction. The denial rate of the candidate solution impact of events and support nodes of the solution that nodes are possessing high risk values or possessing high denial rate then the denial rate of the candidate solutions will be higher impact on the solution $\mathrm{S} 3$

\section{S3 <- G2 G4 G7 G8 G10 G11}

Where G2 G7 and G11 partial denial values can be calculated as the sum of the evidence DEN S3, the risk values are ranging from 3, 2 and 1 for full partial and null denials $\mathrm{S} 3$ can be given by Risk $(\mathrm{S} 3)->2+2+2=6$ since $\mathrm{DEN}(\mathrm{G} 2)=\mathrm{DEN}$ $(\mathrm{G} 7)=\operatorname{DEN}(11)=\mathrm{p}$. the candidate solutions are calculated graph is plotted based on the risk and effort values also with the risk calculation so the incorporation of the risk priority value helps in reducing the level of risk by its priority if the risk calculated as 3 and another risk is calculated as 4 but if the risk value is 3 has a risk priority mapped as high and risk with value 4 is mapped with risk priority low. According to the priority chose risk with value 3 as dominant risk as compared to the risk with value 4 the cost and risk values of the candidate solutions obtained after the GA is plotted.

Table 3 is Candidate effort for requirement analysis

\begin{tabular}{|c|c|c|}
\hline Candidate solutions & cost & Risk \\
\hline$[\mathrm{G} 4, \mathrm{G} 7, \mathrm{G} 8, \mathrm{G} 9, \mathrm{G} 10]$ & 18 & 3 \\
\hline$[\mathrm{G} 4, \mathrm{G} 6, \mathrm{G} 7, \mathrm{G} 8, \mathrm{G} 9]$ & 23 & 6 \\
\hline$[\mathrm{G} 2, \mathrm{G} 7, \mathrm{G} 8, \mathrm{G} 9, \mathrm{G} 10]$ & 19 & 8 \\
\hline$[\mathrm{G} 3, \mathrm{G} 4, \mathrm{G} 5, \mathrm{G} 6, \mathrm{G} 7]$ & 19 & 5 \\
\hline $\begin{array}{c}{[\mathrm{G} 2, \mathrm{G} 3, \mathrm{G} 4, \mathrm{G} 5, \mathrm{G} 6, \mathrm{G} 7,} \\
\mathrm{G} 8]\end{array}$ & 31 & 4 \\
\hline $\begin{array}{c}{[\mathrm{G} 2, \mathrm{G} 4, \mathrm{G} 5, \mathrm{G} 6, \mathrm{G} 7, \mathrm{G} 8,} \\
\mathrm{G} 9, \mathrm{G} 10]\end{array}$ & 31 & 3 \\
\hline
\end{tabular}

The above table shows different candidate solutions with cost and risk value and now the task is to select a candidate solution which effort is effective that risk mapping of the calculated risk values are subjected to fix the above problem, solutions is considered after checking with the risk priority graph. The risk priority generated from the proposed approach based on the risk values of the candidate solutions obtained after the GA candidate solutions [G2, G3,G4, G5, G6,G7 and G8] selected as the effective solution for the software development. 


\section{CONCLUSION}

This analysis presents requirement Engineering of ERP module integration using Tropos Goal Modelling framework alogirthm to analyse risk during the process of evaluation and selection of alternatives. Three layers of goal model to be achieved by the process and events that triggers the goals also support the parameters for the events using genetic algorithm candidate solution, the proposed approach is ERP integration modules limited by considering the association values and event layer. The risk analysis in ERP integration is cost and risk priority calculation carried out on a case study of considering a software development organization, results of the proposed goal model supports for the goal events are plotted.

\section{ACKNOWLEDGMENT}

The work presented in this paper was completed under the guidance of my esteemed guide Prof.Dr.Pabboju Suresh. H.O.D., C.B.I.T.,Hyderabad.I express my deep sense of gratitude towards my guide and all the staff members for their valuable suggestions.

\section{REFERENCES}

[1] Asnar Y, Giorgini P, Modelling risk and identifying countermeasures in organizations. In: Proceedings of the 1st international workshop on critical information infrastructures security, Springer-Verlag, Lecture Notes in Computer Science, vol 4347, pp 55-66, 2006

[2] Anton AI, "Goal-based requirements analysis." In Proceedings of the 2nd IEEE International conference on requirements engineering, IEEE Computer Society Press, pp: 136, 1996.

[3] B. W. Boehm. Software Risk Management: Principles and Practices. IEEE Software, pp.32-41, 1991. [4] Risk Analysis as part of the Requirements Engineering Process YudistiraAsnar Paolo Giorgini March 2007

[4] K.Venkatesh Sharma and Dr P. V. Kumar. "an efficient goal oriented risk analysis in requirement engineering." advance computing conference (iacc), 2013 ieee 3rd international. ieee, feb 2013. this paper is achieved in ieee xplore ISSN : 978-1-4673-4527-9

[5] K.Venkatesh Sharma and Dr P. V. Kumar. "An efficient risk analysis in requirement engineering." engineering (nuicone), dec 2012 nirma university international conference on. ieee, 2012. this paper is achieved in ieee xplore ISSN :978-1-4673-1720-7

[6] K.Venkatesh Sharma and Dr P.V.Kumar "An efficient risk analysis using modified tropos goal model" at international conference on advances in computational intelligence for information technology and communications - ciitcom 2012 in chennai in dec 03 2012. this paper is achieved by springer and is available in springer- digital library ISSN :1867-8211
[7] K.Venkatesh Sharma and Dr P.V.Kumar presented paper on "An efficient risk analysis using modified goal risk model" at fourth international conference on advances in recent technologies in communication and computing ARTCOM 2012 in bangalore in oct 19 2012. this paper is achieved by springer and is available in springer- digital library ISSN: 1876-1100

[8] K.venkatesh Sharma and Dr P. V. Kumar. "Goal oriented analysis for approximate solution using tropos goal model." National conference on Advanced Computing and Pattern Recognition-jan 2014 at JNTUHCEJ ISBN: 9789383038176 pg $78-83$

[9] K.Venkatesh Sharma, Dr P V Kumar "Requirement engineering concepts in risk analysis" (pages 410-414)in ciit International Journal of Software Engineering and Technology" nov 2012 vol 4,no 10 doi : se112012002 impact factor 0.261 ISSN $0974-9748$.

[10] Venkatesh K Sharma and Dr P V Kumar. article: "An efficient risk analysis based risk priority in requirement engineering using modified goal risk model". International Journal of Computer Applications 73(14):15-24, july 2013. published by Foundation of computer science, New York,USA. bibtex doi: 10.5120/12808-9939 impact factor 0.821 ISSN: 0975 8887.

[11] Venkatesh K Sharma and Dr P V Kumar. article: "A method to risk analysis in requirement engineering using tropos goal model with optimized candidate solutions" in. International Journal of Computer Science Issues, issue 6, nov 2013 impact factor 0.242 ISSN 1694-0814.

[12] Venkatesh K Sharma and Dr P V Kumar. article "A method to risk analysis in requirement engineering through optimized goal selection tropos goal layer" in journal of Theoretical and Applied information technology 20 march 2014 -- vol. 61. no. 2 - 2014 ISSN: 19475500 , impact factor 0.423

[13] JacKyAng, Sook Bing Leong, Chin Fei Lee, UmiKalsomYusof, "Requirement Engineering Techniques in Developing Expert Systems", School of Computer Sciences UniversitiSains Malaysia, IEEE, symposium on computer science and informatics, 2011, pp.1-2

[14] Lukas Pilat and Hermann Kaindl, "A Knowledge Management Perspective of Requirements Engineering", Institute of Computer Technology Vienna University of Technology Vienna, Austria, IEEE Conference: 19-21 May 2011, pp.1-12.

[15] Mina Attarha and Nasser Modiri, "Focusing on the Importance and the Role of Requirement Engineering", Interaction Sciences (ICIS), 2011 4th International Conference, 16-18 Aug. 2011, pp. $181-184$. 